\title{
Sustainable Architecture: the Eco-Efficiency Earth Construction
}

\author{
Cesare Sposito ${ }^{1}$, Francesca Scalisi ${ }^{2}$
}

\begin{abstract}
:
The appropriate choice of building materials can contribute decisively to reduce the energy consumption of the building sector. The increasing worries for the environmental impact of construction materials is witnessed by a lot of studies The mentioned worries has brought again the attention towards natural materials. The use of more sustainable construction materials and construction techniques represent a major contribution to the eco-efficiency of the construction industry and thus to a more sustainable development.

Within this frame the renewed interest for a natural material such as the rammed earth is included; the interest has been in continuous growth from the 80 s till today.

The rammed earth's advantages regarding these requirements are evident: the resource is available in great amounts, the energy needed for extraction, transformation and production of the material is very low and it is a wholly recyclable material. Besides these benefits, the interest is due to its high thermal inertia and hard hygroscopic properties.
\end{abstract}

Keywords: earth construction, sustainability, architecture

\section{Introduzione ${ }^{1}$}

Secondo la definizione della Bruntland Commission, lo sviluppo sostenibile consiste nel cercare di soddisfare i bisogni del presente senza compromettere la possibilità delle genera- zioni future di soddisfare i propri bisogni [1]. Il termine sostenibilità, utilizzato inizialmente per definire più eque dinamiche di sviluppo economico, è rapidamente diventato di uso comune ed è stato via via impiegato per caratterizzare non solo il modello di sviluppo, ma anche le singole azioni o situazioni in cui tale modello si applica e si concretizza, da produzione sostenibile a mercato sostenibile, fino a edificio sostenibile (sustainable building): un edificio a cui si chiede di evitare l'impiego di materiali inquinanti, di privilegiare i materiali organici naturali, di utilizzare elementi riciclati, di limitare il ricorso a combustibili fossili, di ridurre la produzione di rifiuti e il consumo energetico nella fase di esercizio.

Il settore dell'edilizia ha da diverso tempo mostrato un particolare interesse per le questioni legate alla sostenibilità ambientale e all'efficienza energetica, non solo attraverso l'utilizzo di tecnologie e materiali innovativi, ma anche con la reintroduzione di sistemi di costruzione che utilizzano materiali naturali e tradizionali. L'impiego della terra cruda come materiale da costruzione contemporaneo risponde a questi requisiti. Costruire con la terra significa ripensare, sia a livello locale sia a livello globale, l'impiego delle risorse del nostro pianeta; difendere il diritto di mettere in opera un materiale da costruzione naturale ed ecologico, abbondante, facilmente disponibile e accessibile al 
maggior numero possibile di persone; promuovere le risorse locali, sia umane che naturali, il miglioramento delle condizioni di vita, valorizzare la diversità culturale e mantenere sistemi di sostegno sociale per la costruzione e la manutenzione dell'ambiente costruito; utilizzare un calcestruzzo naturale, che offra una vera alternativa ecologica ed economica rispetto a materiali e processi produttivi nocivi per l'ambiente. L'uso della terra cruda nelle costruzioni vanta una tradizione millenaria che si è sviluppata in tutte le aree geografiche: secondo Berge [2] ed altri studiosi [3] le testimonianze più antiche sono da ricercarsi nella Valle del Tigri, risalenti al 7500 a.C.; la Grande Muraglia cinese la cui costruzione iniziò circa 3000 anni fa, presenta ampie sezioni costruite in terra cruda così come il Tempio Horyuji in Giappone risalente a 1300 anni fa [4]; le rovine della città di Chanchán in Perù e il villaggio di Taos nel New Mexico sono tra le testimonianze più antiche di costruzioni in terra cruda [5]; i resti delle fortificazioni greche di capo Soprano a Gela in Sicilia [6-8], risalenti al sec. IV a.C. note anche per la presenza di muri in terra cruda (Fig. 1) o le città mediorientali interamente realizzate in terra, come Shibam nello Yemen (Fig. 2), costruita nel sec. XVI [9].

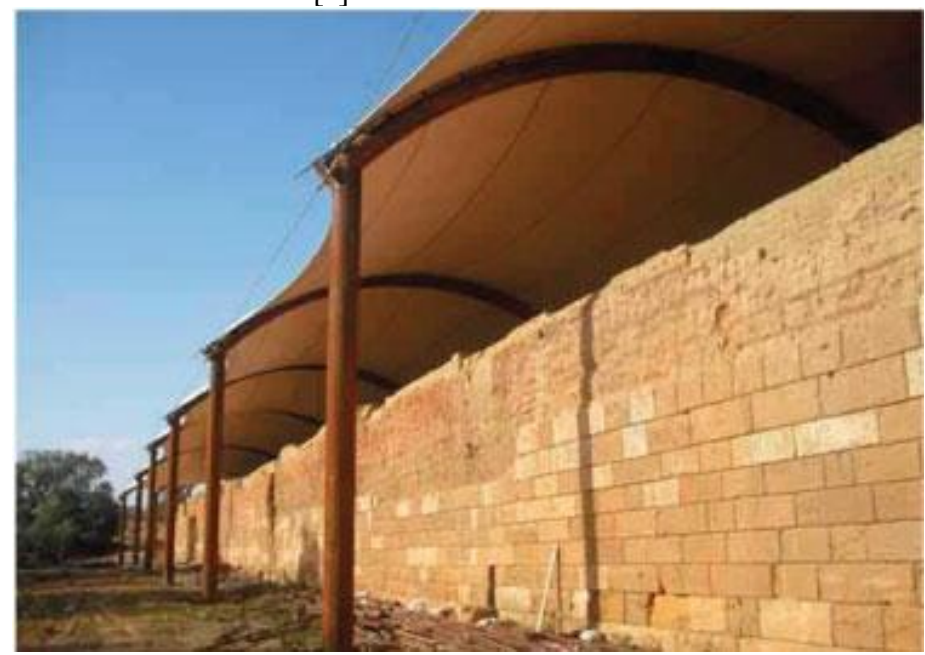

Fig. 1 - Le fortificazioni di Capo Soprano a Gela del sec. IV a.C.

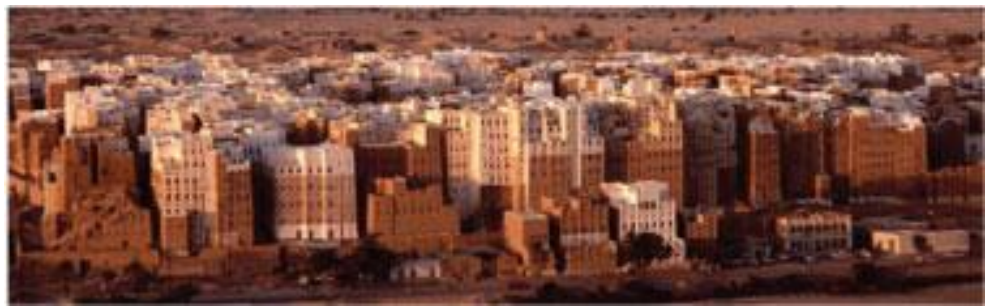

Fig. 2 - La città di Shibam nello Yemen del sec. XVI.

\section{Terra cruda e risparmio energetico ${ }^{1}$}

Il settore delle costruzioni, a livello globale, contribuisce per il 30\% all'emissione di anidride carbonica, consumando più materie prime rispetto a qualsiasi altra attività 
economica; un andamento che dimostra come il settore sia insostenibile e necessiti di scelte che inneschino una inversione di tendenza. In più, l'aumento della popolazione mondiale e la conseguente maggiore richiesta di edifici e infrastrutture, potrebbero ulteriormente fare aumentare il consumo di materiali non rinnovabili, così come la produzione di rifiuti. Pertanto, l'uso di materiali da costruzione più sostenibili rappresenta un importante contributo alla eco-efficienza del settore delle costruzioni e, quindi, a uno sviluppo più sostenibile. Dato che l'edilizia consuma in tutto il suo ciclo di vita oltre il 40\% di tutta l'energia prodotta [10], è necessario intervenire per ridurre drasticamente tale consumo, in particolare la scelta di materiali costruttivi più sostenibili può incidere in maniera significativa sulla riduzione del consumo di energia [11] e sulla riduzione delle emissioni di $\mathrm{CO}_{2}$ in atmosfera [12].

Il fabbisogno energetico di un edificio è la somma dell'energia necessaria per il riscaldamento, il raffrescamento, la ventilazione e l'illuminazione. La somma di tali consumi incide fortemente sul bilancio energetico nazionale e, alla riduzione di tale incidenza, sono dedicate numerose ricerche. Alcune di queste sono tese all'individuazione di nuove fonti energetiche rinnovabili, altre alla riduzione dei consumi attraverso l'efficientamento dell'involucro degli edifici e al miglioramento degli impianti. Tutte queste ricerche mirano all'efficientamento del consumo energetico nella fase di esercizio degli edifici, misurando l'Operating energy [13].

La progettazione di un edificio a energia quasi zero deve affrontare anche la scelta e l'impiego di materiali che, nelle fasi di produzione, messa in opera e dismissione mostrino un ridotto consumo di energia e una ridotta quantità di emissioni in atmosfera: l'Embodied energy e l'Embodied carbon, sono intesi come il totale dell'energia primaria impiegata (o dell'anidride carbonica emessa) durante le fasi del ciclo di vita del bene [1415].

I vantaggi della terra cruda rispetto a tali esigenze sono evidenti: la risorsa è disponibile in grandi quantità, l'energia necessaria per estrarre, trasformare e produrre la terra cruda è estremamente bassa ed è un materiale totalmente riciclabile; di contro non presenta un'elevata resistenza meccanica e non può essere utilizzato in maniera indiscriminata. Realizzare mattoni in terra non richiede una grande quantità di energia: infatti, a differenza dei comuni laterizi, non hanno bisogno della fase di cottura (durante la quale viene emessa una grande quantità di $\mathrm{CO}_{2}$ ), ma sono semplicemente essiccati in maniera naturale.

Studiosi come Morton [16], Lourenço [17] e Shukla [18] hanno condotto studi che confrontano le emissioni di anidride carbonica di edifici realizzati in terra cruda con edifici realizzati con murature tradizionali concludendo che l'energia incorporata utilizzata negli edifici in terra cruda è notevolmente inferiore rispetto a quella utilizzata per quelli in muratura tradizionale.

Oltre a questi vantaggi, il principale interesse di questo materiale certamente sta nella sua elevata inerzia termica e nelle sue forti proprietà igroscopiche.

Nella fase di esercizio, i mattoni in terra aumentano il comfort dell'edificio e contribuiscono al risparmio energetico, in quanto:

- regolano l'umidità perché possono mantenere costantemente un grado di umidità dell'aria intorno al 50\%; livelli di umidità superiore al 70\% sono responsabili della comparsa di muffe che possono innescare reazioni allergiche [19], e valori di 
umidità relativa superiore al $60 \%$ sono associati con la presenza di acari e malattie asmatiche [20]; di contro, una umidità relativa inferiore al 40\% determina un'aria interna molto secca che causa malattie respiratorie;

- regolano la temperatura: grazie alla loro massa elevata $(1800 \mathrm{~kg} / \mathrm{m} 3)$, fanno da volano termico, impedendo gli sbalzi di temperatura in casa; il calore che entra dalle finestre, quello prodotto dall'uomo, dagli elettrodomestici e dalle luci, viene assorbito dalla terra, che lo cede nuovamente quando necessario, ad esempio la sera; ciò aumenta il comfort domestico e favorisce notevolmente il risparmio energetico;

- isolano dal caldo: grazie alla loro massa elevata e al calore specifico, impediscono al caldo di entrare; di notte l'ambiente si rinfresca, e al mattino, quando arriva il caldo, i mattoni in terra assorbono di nuovo una grande quantità di calore, riducendo così la temperatura degli ambienti durante il giorno;

- isolano dal rumore: $\mathrm{i}$ mattoni in terra hanno ottimi valori di abbattimento acustico, perché essendo realizzati con un materiale "elastico" assorbono i rumori impedendogli di passare;

- proteggono dall'inquinamento elettromagnetico: $15 \mathrm{~cm}$ di terra abbattono ben il 99\% delle onde elettromagnetiche, un valore superiore a tutti gli altri materiali da costruzione;

- purificano l'aria: l'odore in casa viene trasportato dal vapore acqueo; la terra, assorbendo il vapore acqueo, funge da filtro naturale e purifica l'aria;

- evitano la condensa: proprio perché in grado di assorbire l'umidità, i mattoni in terra impediscono la formazione di condensa nel muro, anche quella interstiziale.

\section{Architettura contemporanea in terra cruda ${ }^{2}$}

La fede assoluta nel calcestruzzo armato, che ha attraversato il secolo scorso, ha oscurato le enormi potenzialità della terra cruda come materiale da costruzione. Ma in un'epoca in cui abbiamo cominciato a prendere coscienza dei limiti che hanno le risorse del nostro pianeta, la terra cruda viene sempre più spesso considerata il materiale ecologico per eccellenza.

La terra è un materiale da costruzione che, in virtù della sua versatilità, è in grado di porsi perfettamente in equilibrio con l'ambiente: la terra si presta a realizzazioni accessibili a qualsiasi organizzazione produttiva, dalla più semplice alla più complessa; inoltre è una risorsa facilmente reperibile nella gran parte dei contesti geografici e la varietà delle prestazioni ottenibili dalle diverse tecniche esecutive consente di produrre manufatti adatti a perseguire il risparmio energetico e il comfort in differenti contesti climatici.

L'adobe è uno dei prodotti più diffusi tra quelli riconducibili alla terra cruda (Fig. 3): si tratta di un mattone di terra cruda formato a mano, con stampo, senza compressione e lasciato seccare naturalmente; tra $i$ più impiegati, soprattutto in epoca recente, vanno ricordati il blocco compresso (Fig. 4), un mattone compresso con l'ausilio di presse meccaniche per aumentare le caratteristiche di compressione, e il blocco estruso, la cui esecuzione è simile a quella dei laterizi. Un'altra tecnica costruttiva molto diffusa è il pisé (Fig. 5), che consiste nella formatura e compressione della terra in 
apposite casseforme: il materiale viene inserito in strati di $\mathrm{cm} \mathrm{5-12} \mathrm{e} \mathrm{battuto} \mathrm{fino} \mathrm{ad}$ arrivare a strati di circa $\mathrm{cm} 80$.
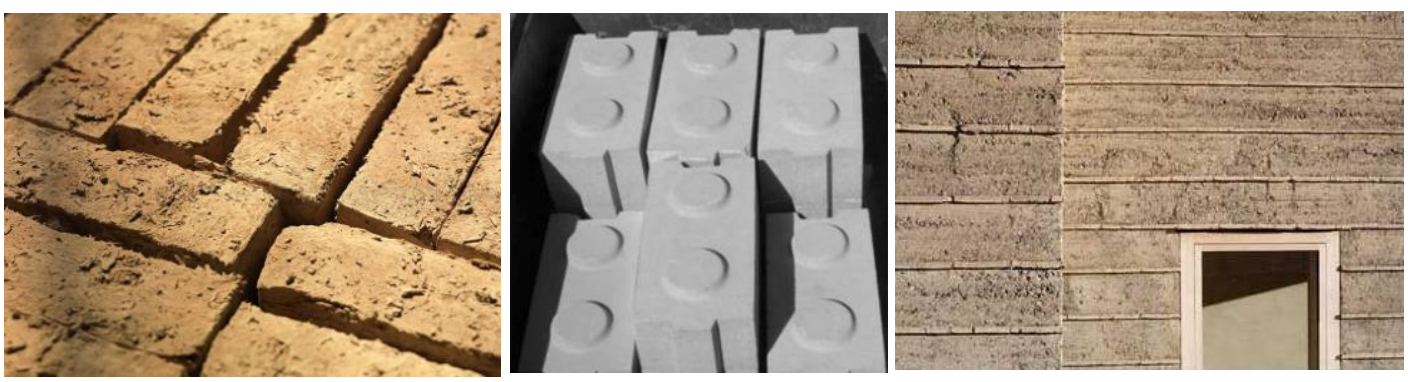

Figg. 3, 4, 5-Adobe (a sinistra); blocchi compressi (al centro); muro in pisé (a destra).

L'utilizzo della terra cruda ha interessato svariati tipi edilizi come musei, scuole, alberghi, ville, soprattutto in Paesi come l'Australia, il Canada o gli Stati Uniti, dove esistono già da tempo diversi regolamenti sulle costruzioni in terra cruda.

In Canada si trova il NK'MIP, un centro culturale nel deserto della Columbia Britannica (Fig. 6). L'area in cui sorge l'edificio è di proprietà degli indiani Osoyoos e rappresenta un habitat unico nel panorama canadese. Il progetto iniziale, di Hotson Bakeker Boniface Haden Architects + Urbanistes $(\mathrm{HBBH})$, era costituito da un edificio in cemento a cui successivamente è stato aggiunto un lungo muro in pisé. Il muro si integra perfettamente con l'ambiente circostante e soddisfa anche le esigenze della comunità indiana, che stentava a riconoscersi in un edificio in cemento. L' impasto è stato realizzato con terra locale e coloranti uniti a una percentuale di cemento di poco superiore al $6 \%$.

In Australia svolge la sua attività l'architetto Alan Powell, che nel 2004 ha realizzato a Victoria un museo in terra, il The TarraWarra Museum of Art (Fig. 7). Nelle aree desertiche degli Stati Uniti l'integrazione con il paesaggio circostante può essere realizzata soltanto con l'utilizzo di materiali naturali come la terra. A Tucson, in Arizona, l'architetto Rick Joy ha progettato la casa Palmer-Rose con muri in pisé (Fig. 8), la stessa tecnica impiegata per l'ingresso al McDowell Sonoran Preserve. Sempre in Arizona, a Scottsdale, è la Dirt House (1997), un edificio residenziale ad un unico livello, opera dell'architetto Neil Jones [21].

In Europa, Martin Rauch è considerato uno dei pionieri delle moderne tecniche costruttive per l'applicazione di tradizionali metodi di costruzione in terra. L'architetto austriaco ha realizzato un volume in argilla nella Chiesa della Conciliazione a Berlino (Fig. 9), con una parete spessa $\mathrm{cm} 60$ e alte $\mathrm{m}$ 7. L'edificio sorge sulle rovine della vecchia Chiesa eretta nel 1894, caduta in rovina negli anni successivi alla costruzione del Muro di Berlino; il nuovo progetto è composto da due corpi edilizi ovali, collocati uno dentro l'altro ma con gli assi principali ruotati ortogonalmente: l'ovale esterno è realizzato in lamelle di legno mentre quello interno è in argilla compressa. La Cappella della Riconciliazione, inaugurata nel 2000, rappresenta la prima chiesa tedesca in argilla compressa portante. 

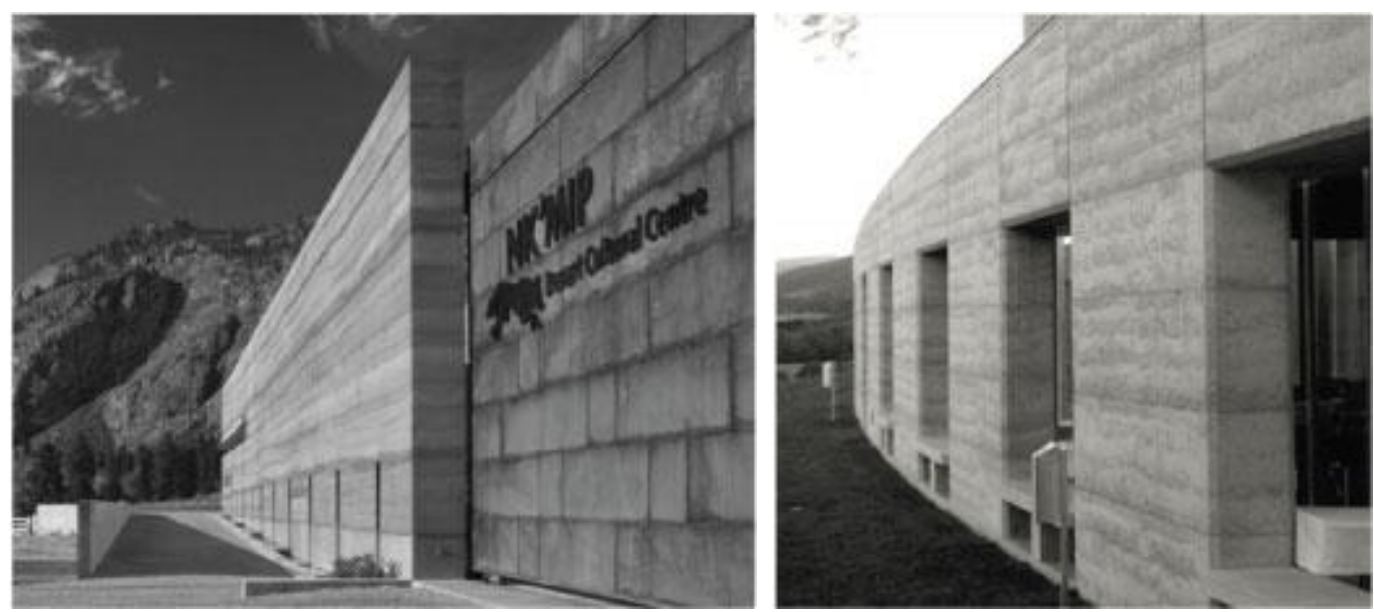

Figg. 6, 7 - Il muro in pisè del Nk'mip Cultural Centre in Canada (a sinistra); il TarraW arra Museum of Art in Australia (a destra).
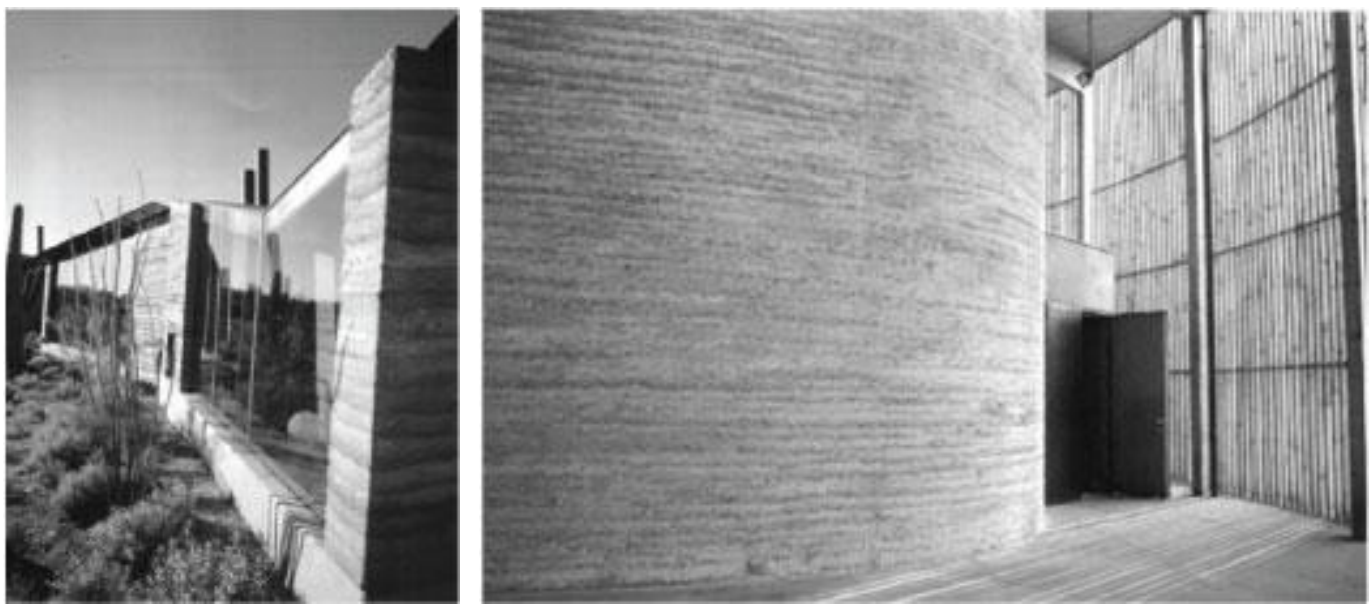

Figg. 8, 9 - La casa Palmer-Rose in Arizona (a sinistra); La Chiesa della Riconciliazione a Berlino (a destra).

Nel 2008 Martin Rauch ha realizzato a Schlins, in Austria, la propria abitazione, insieme con l'architetto Roger Boltshauser. Si tratta di un edificio di tre livelli, realizzato in terra compressa stratificata, con sottili fila di mattoni disposti a intervalli regolari, visibili nella facciata. Le pareti, i pavimenti e gli intonaci sono realizzati completamente con la terra dello sbancamento, risultato della volontà di costruire una casa esclusivamente con materiali sostenibili. La casa in terra ha una struttura monolitica, si presenta come un blocco scultoreo, una natura astratta e artificiale spinta verso l'alto dalla terra sottostante. Già nel 1994 Martin Rauch aveva costruito il suo Atelier in terra cruda sempre a Schlins, diventato un edificio di riferimento per gli operatori del settore. Non da meno è l'opera dell'architetto Minke [22], di cui ricordiamo la costruzione del Giardino d'infanzia Waldorf a Sorsum, in Germania, realizzato in blocchi di terra estrusi. 


\section{L'attuale ricerca sulla terra cruda ${ }^{2}$}

La terra cruda ha ricevuto nell'ultimo decennio una grande attenzione da parte della comunità scientifica, come testimonia l'incremento delle ricerche pubblicate nelle riviste di settore.

Come evidenziato da Pacheco-Torgal [23], negli ultimi dieci anni quasi un centinaio di articoli riferiti a questo argomento sono stati pubblicati in riviste Scopus. Se è vero che tale quantità rappresenta solo una piccola parte rispetto agli articoli pubblicati sul cemento Portland nello stesso periodo è anche vero che essa rappresenta un aumento di dieci volte rispetto agli articoli relativi alla terra cruda pubblicati nel 1990.

L'impasto di argilla e acqua costituisce la base per la produzione di mattoni in terra cruda, ma comunemente vengono aggiunti altri elementi, come fibre naturali o cementi, utili a stabilizzare il composto. Stabilizzare una terra significa modificare le proprietà di un sistema terra-acqua per migliorarne le caratteristiche fisico-chimiche e meccaniche.

Dagli articoli pubblicati, emerge che sono molti gli studi condotti sulla resistenza meccanica dei mattoni in terra cruda [24-25] e soprattutto sulla stabilizzazione del materiale, con un'attenzione prevalente per il cemento come stabilizzante [26-28], anche se l'uso del cemento per stabilizzare il materiale incide sull'impatto ambientale in termini di elevato consumo di energia; di recente, sono stati condotti studi anche sulla possibilità di utilizzare il metacaolino (caolino dopo trattamento termico) come stabilizzante, attraverso l'impiego di nanotecnologie. Il caolino viene considerato in alcuni casi un nanomateriale essendo formato da "fogli" di spessore nanometrico sovrapposti [29-30]; diversi sono gli studi condotti anche sulle proprietà igrotermiche del materiale, anche se in misura minore rispetto a quelli sopra indicati [31-32].

Fino ad oggi è stato poco indagato il possibile apporto della nanotecnologia per incrementare le prestazioni della terra cruda, soprattutto se si considera che le nanotecnologie stanno rivoluzionando il settore dei materiali da costruzione e sono considerate determinanti per l'efficienza energetica degli edifici [33]. La nanotecnologia può essere definita come la progettazione, la caratterizzazione, la produzione e l'applicazione di strutture, dispositivi e sistemi controllati alla scala nanometrica (un nanometro equivale ad un miliardesimo di metro).

In questo filone si inserisce il progetto di ricerca sull'uso della LAPONITE® (nanodischi di argilla dello spessore di $1 \mathrm{~nm}$ e il diametro di $25 \mathrm{~nm}$ ) come stabilizzante per migliorare la resistenza meccanica della terra cruda [34-35]. La sperimentazione ha riguardato l'esecuzione di diverse tipologie di adobe: un primo gruppo comprendeva tre tipologie gli adobe tradizionale realizzati rispettivamente con un impasto di terra e acqua, terra, acqua e sabbia, terra, acqua, sabbia e paglia; un secondo gruppo comprendeva le tre tipologie di adobe realizzate rispettivamente con un impasto di terra, acqua e LAPONITE®, terra, acqua, sabbia e LAPONITE®, terra, acqua, sabbia, paglia e LAPONITE®.

Tutte le tipologie di adobe realizzate sono state sottoposte a prove di resistenza a compressione e a flessione, prove di abrasione, prove di resistenza all'urto, prove di assorbimento per capillarità. Il confronto dei risultati tra gli adobe tradizionali e quelli con LAPONITE $\AA$ ha evidenziato le migliori prestazioni degli adobe con nanodischi, 
soprattutto nella resistenza a compressione. Ciò apre sicuramente nuovi ambiti di ricerca che si presentano alquanto promettenti.

\section{Conclusioni $^{2}$}

L'uso della terra cruda è associato a una bassa energia incorporata, basse emissioni di anidride carbonica e ridotto impatto ambientale, prestazioni che giustificano il grande attivismo nel mondo intorno alle costruzioni in terra cruda. La diffidenza nei confronti di questo materiale è ancora molta: anche se le problematiche che le costruzioni in terra cruda presentano sono molteplici e sicuramente la sua utilizzazione non può essere indiscriminata, tali problematiche possono essere superate ponendo l'attenzione su tre aspetti: quello culturale, quello normativo e quello che è riferibile all'incremento della ricerca. L'aspetto culturale e quello relativo alla ricerca si stanno affrontando in maniera crescente, come dimostrano le ricerche e le pubblicazioni che sempre più negli ultimi anni incrementano il corpus bibliografico. Il problema fondamentale rimane la mancanza di Normative di riferimento che siano di supporto agli operatori del settore. Molti paesi comunque hanno iniziato da tempo riflessioni sui vantaggi (economici, ecologici, energetici) di questi sistemi costruttivi; ad esempio, attualmente negli Stati Uniti la costruzione in adobe è integrata nei codici di costruzione nazionali (Uniform Building Code Standards) e dal 1983 alcuni nazioni hanno regolamentato la costruzione in pisé, in blocchi compressi e in zolle. L'Italia è tra i Paesi che non possiede ancora una Normativa per le costruzioni in terra cruda, anche se alcune regioni hanno avviato un processo di tutela speciale per il recupero e la messa in valore del patrimonio di terra. La strada da percorrere è ancora lunga, ma di certo le prospettive di ricerca sono molte e si prefigurano di grande interesse.

\section{References}

[1] WCED (1987). Our Common Future.

[2] Berge, B. (2009). The ecology of building materials. 2 ed. Architectural Press, Elsevier Science.

[3] Pollock, S. (1999). Ancient mesopotamia. Cambridge: University Press.

[4] Jaquin, PA. (2008). Analysis of historic rammed earth construction. PhD thesis. Durham Universit, United Kingdom.

[5] Alexandra, S. (2006). Architecture and earth construction. In Piaui, Research, characterization and analysis. Master thesis, Federal University of Piauí, Brasil.

[6] Adam, J. P. (1982). Greek military architecture. Paris: Picard.

[7] Martin, R. et al. (1979). The Greek cities. Gela. In Gabba, E., Vallet, G. (Eds.). History of Sicily, I, 3. Napoli: Società Editrice History of Naples and Sicily.

[8] Panvini, R. (1996). Gela. History and archeology of the ancient Gela. Torino: SEI.

[9] Helfritz, H. (1937). Land without shade. J Roy Central Asian Soc, 24(2), 201-16.

[10] OCDE (2003). Environmental sustainable building - challenges and policies. Paris, France, p. 194.

[11] Thormark, C. (2006). The effect of material choice on the total energy need and recycling potential of a building. Build Environ, 41, 1019-26.

[12] Gonzalez, M, Navarro, J. (2006). Assessment of the decrease of $\mathrm{CO}_{2}$ emissions in the construction field through the selection of materials. Build Environ, 41, 902-9.

[13] Barucco, M.A., Verde, F., Scalisi, F. (2016). Innovazione tecnologica di sistemi, componenti e materiali/Technological innovation of systems, components and materials. In Lucarelli, M.T., Mussinelli, E., Trombetta, C. (eds). Cluster in progress. La Tecnologia dell'arcbitettura in rete per 
l'innovazione / The Architectural technology network for innovation. Santarcangelo di Romagna (RM): Maggioli, 103-108.

[14] Geoff, H., Craig, J. (2008). Inventory of Carbon \& Energy (Ice), version 1.6a. Proc. Inst. Civil Engrs.: Energy, University of Bath.

[15] Hammond, G. P. and Jones, C. I. (2008). Embodied energy and carbon in construction materials. Proceedings of the Institution of Civil Engineers - Energy, 161 (2), 87-98.

[16] Morton, T., Stevenson, F., Taylor, B., Smith, C. (2005). Low cost earth brick construction. Monitoring and evaluation. Arc. Architects.

[17] Lourenço, PI. (2002). Earth constructions. Master thesis, UTL-IST, Lisbon, Portugal.

[18] Shukla, A., Tiwari, G., Sodha. MS. (2008). x Embodied energy analysis of adobe house, Renew Energy, 34, 75561.

[19] Arundel, A., Sterling. E, Biggin. J, Sterling. T. (1986). Indirect health effects of relative humidity in indoor environments. Environ Health Perspect, 65, 351-61.

[20] Howieson, S. (2005). Housing and asthma. London: Spon Press.

[21] Rael, R. (2008). Earth Architecture. New York: Princeton Architectural Press.

[22] Minke, G. (2000). Earth construction handbook. The building material earth in the modern architecture. Southampton, UK: WIT Press.

[23] Pacheco-Torgal, F., Jalali, S. (2012). Earth construction: Lessons from the past for future eco- efficient construction. Construc Build Mater, 29, 512-9.

[24] Mbereyaho, L., Twubahimana, J. and Mureramanzi, G. (2014). Strength characteristics of earth bricks and their application in construction. International Research Journal on Engineering, 2(1), 001-007.

[25] Morel, JC., Pkla, A., Walker, P. (2007). Compressive strength testing of compressed earth blocks. Construc Build Mater, 21, 303-9.

[26] Millogo, Y., Morel, JC. (2012). Microstructural characterization and mechanical properties of cement stabilised adobes. Mater Struct, 45, 1311-8.

[27] Ciancio, D., Gibbings, J. (2012). Experimental investigation on the compressive strength of cored and molded cement-stabilized rammed earth samples. Construc Build Mater, 28, 294-304.

[28] Venkatarama Reddy, BV., Prasanna Kumar, P. (2011). Cement stabilised rammed earth. Part B: Compressive strength and stress-strain characteristics. Mater Struct, 44, 695-707.

[29] Maskell, D., Heath, A. and Walker, P. (2015). Use of Metakaolin with stabilised extruded earth masonry units. Construc Build Mater, 78(1), 172.

[30] Niroumanda, H., Zainb, MFM., Alhosseinic, SN. (2013). The Influence of Nano-Clays on Compressive Strength of Earth Bricks as Sustainable Materials. Procedia - Social and Behavioral Sciences, 89, 862-865.

[31] Maillard, P., Aubert, JE. (2014). Effects of the anisotropy of extruded earth bricks on their hygrothermal properties. Construc Build Mater, 63, 56-61.

[32] Cagnon, H. et al. (2014). Hygrothermal properties of earth bricks. Energy and Buildings, 80, 208-217.

[33] Ashby, MF., Ferreira, PJ., Schodek, DL. (2009). Nanomaterials, nanotechnologies and design. Oxford: Elsevier.

[34] Scalisi, F., Sposito, C. (2017) Performance of Earth Bricks supplemented by Nanoparticles and their application in Architecture. Applied Mechanics and Materials, 864, 8-13.

[35] Scalisi, F. (2014) Nanotechnology and earth construction: the mechanical properties of adobe brick stabilized by Laponite nanoparticles. Advansed Materials Recearch, 983, 63-66.

1 Paragrafo curato da Francesca Scalisi.

2 Paragrafo curato da Cesare Sposito. 